\title{
A NONLOCALLY CONNECTED CONTINUUM $X$ SUCH THAT $C(X)$ IS A RETRACT OF $2^{X}$
}

\author{
JACK T. GOODYKOONTZ, JR.
}

\begin{abstract}
Let $X$ be a metric continuum and let $2^{X}(C(X))$ denote the hyperspace of closed subsets (subcontinua) of $X$. An example is given of a nonlocally connected continuum $X$ such that $C(X)$ is a retract of $2^{X}$.
\end{abstract}

By a continuum we mean a compact connected metric space. If $X$ is a continuum, then $2^{X}$ (respectively, $C(X)$ ) denotes the hyperspace of closed subsets (respectively, subcontinua) of $X$, each with the Hausdorff metric. In this note we will obtain a partial answer to a question raised by Sam B. Nadler, Jr. [8] by giving an example of a nonlocally connected continuum $X$ such that $C(X)$ is a retract of $2^{X}$.

In 1939 Wojdyslawski [10] proved that $C(X)$ is an absolute retract if and only if $X$ is locally connected. Hence, $C(X)$ is a retract of $2^{X}$ when $X$ is locally connected. In Theorem 3.6 of [8], Nadler shows that $C(X)$ is always a continuous image of $2^{X}$. Following this result, in Question 3.7 of [8], Nadler asks, "When is $C(X)$ a retract of $2^{X}$ ?" Nadler discusses this question further in [6] and in Chapter 6 of [7]. In particular, in Theorem 6.11 of [7], Nadler proves a result which indicates that, when $X$ is not locally connected, a retraction from $2^{X}$ onto $C(X)$, if one exists, must be rather complicated. In [2] the author determined the first partial answer to Nadler's question by giving an example of a nonlocally connected continuum $X$ such that $C(X)$ is not a retract of $2^{X}$. In [5] Lawson attempted to give some conditions which would imply the existence of a retraction from $2^{X}$ onto $C(X)$ for certain nonlocally connected continua. However, as noted in [2], Lawson's proof was not correct. We will show that for one of the examples in Lawson's paper, namely the cone over a convergent sequence, there does exist a retraction from $2^{X}$ onto $C(X)$. It is interesting to note that this example and the example in [2] are both smooth dendroids. Thus, even within this rather well-behaved class of continua, $C(X)$ may or may not be a retract of $2^{X}$ when $X$ is not locally connected.

We observe in Corollary 1 that the example in this paper also has the property that $F_{1}(X)$, the space of singleton subsets of $X$, is a retract of $2^{X}$. This is the first example of a nonlocally connected continuum for which it has been shown that $F_{1}(X)$ is a retract of $2^{X}$. Hence, the example also gives a partial answer to Nadler's question in 6.2 of [7].

Received by the editors July 5, 1983 and, in revised form, September 8, 1983.

1980 Mathematics Subject Classification. Primary 54B20; Secondary 54C15.

Key words and phrases. Continuum, hyperspace, retract, smooth dendroid. 
The symbol $I$ will denote the closed unit interval of real numbers and the symbol $\mathbf{Z}^{+}$will denote the set of positive integers. For the remainder of this paper, the symbol $X$ will denote the specific continuum defined in the following paragraph. We will let $d$ denote the usual Euclidean metric on $X$ and $\rho$ denote the Hausdorff metric on $2^{X}$. An order $\operatorname{arc} \beta$ in $C(X)$ is an arc with the property that whenever $A, B \in \beta$, then $A \subset B$ or $B \subset A$.

We describe the planar continuum $X$ in terms of polar coordinates. Let $T=\{0\}$ $\cup\left\{1 / n \mid n \in \mathbf{Z}^{+}\right\}$. For each $\theta \in T$, let $L_{\theta}=\{(r, \theta) \mid r \in I\}$. Let $X=\bigcup\left\{L_{\theta} \mid \theta \in T\right\}$, $p=(0,0), 2_{p}^{X}=\left\{A \in 2^{X} \mid p \in A\right\}$, and $\mathscr{A}=2_{p}^{X} \cup\left(\left\{2^{L_{\theta}} \mid \theta \in T\right\}\right)$. We note that $\mathscr{A}$ is closed and that $C(X) \subset \mathscr{A}$.

We now list all of the functions that we use to define a retraction $R$ from $2^{X}$ onto $C(X)$. Define mappings $r_{\min }, r_{\max }: 2^{X} \rightarrow I$ by

$$
r_{\min }(A)=\min \{r \mid(r, \theta) \in A\} \quad \text { and } \quad r_{\max }(A)=\max \{r \mid(r, \theta) \in A\} .
$$

Define mappings $\theta_{\min }, \theta_{\max }: 2^{X}-2_{p}^{X} \rightarrow I$ by

$$
\theta_{\min }(A)=\min \{\theta \mid(r, \theta) \in A\} \quad \text { and } \quad \theta_{\max }(A)=\max \{\theta \mid(r, \theta) \in A\} .
$$

Define the mapping $g: 2^{X}-2_{p}^{X} \rightarrow C(X)$ by

$$
g(A)=\left\{\left(r, \theta_{\min }(A)\right) \mid r_{\min }(A) \leqslant r \leqslant r_{\max }(A)\right\} .
$$

Define the function $f: 2^{X} \rightarrow C(X)$ by

$$
f(A)=\bigcap\{M \in C(X) \mid A \subset M\} .
$$

Using Theorem 1 of [1] and Theorems 1,2 and 3 of [4], it is easy to show that if $A \in 2^{X}$ and $A \not \subset L_{0}-\{p\}$, then $f$ is continuous at $A$. Define the mapping $\alpha$ : $\left(2^{X}-\mathscr{A}\right) \times I \rightarrow C(X)$ by

$$
\alpha(A, s)= \begin{cases}h_{1}(g(A), 3 s) & \text { if } s \in\left[0, \frac{1}{3}\right], \\ f(\{p\} \cup g(A)) \cup h_{2}(A, 3 s-1) & \text { if } s \in\left[\frac{1}{3}, \frac{2}{3}\right], \\ f(A) \cup h_{2}\left(\left\{\left(r_{\max }(A), \theta_{\min }(A)\right)\right\}, 3-3 s\right) & \text { if } s \in\left[\frac{2}{3}, 1\right],\end{cases}
$$

where $h_{1}$ and $h_{2}$ are as defined on p. 36 of [3]. If $A \in 2^{X}-\mathscr{A}$, then $\{\alpha(A, s) \mid s \in$ $\left.\left[0, \frac{1}{3}\right]\right\}$ is the unique order arc in $C(X)$ from $g(A)$ to $f(\{p\} \cup g(A)),\{\alpha(A, s) \mid s \in$ $\left.\left[\frac{1}{3}, \frac{2}{3}\right]\right\}$ is the order arc in $C(X)$ which grows uniformly from $f(\{p\} \cup g(A))$ to $f(\{p\} \cup g(A)) \cup f(A)$, and $\left\{\alpha(A, s) \mid s \in\left[\frac{2}{3}, 1\right]\right\}$ is the unique order arc (possibly degenerate) in $C(X)$ from $f(A)$ to $f(\{p\} \cup g(A)) \cup f(A)$. Thus, for each $A \in 2^{X}-$ $\mathscr{A},\{\alpha(A, s) \mid s \in I\}$ is an arc in $C(X)$ from $g(A)$ to $f(A)$. Define the function $R$ : $2^{X} \rightarrow C(X)$ by

$$
R(A)= \begin{cases}f(A) & \text { if } A \in \mathscr{A}, \\ \alpha\left(A, \min \left\{\theta_{\max }(A) / r_{\min }(A), 1\right\}\right) & \text { if } A \in 2^{X}-\mathscr{A} .\end{cases}
$$

THEOREM 1. Let $R$ be as defined in (1). Then $R$ is a retraction from $2^{X}$ onto $C(X)$.

Proof. It is clear that $R$ is continuous on the open set $2^{X}-\mathscr{A}$. Let $A \in \mathscr{A}$. If $A \in 2_{p}^{X}$, then $f$ is continuous at $A$. If $A \in \mathscr{A}-2_{p}^{X}$, then $f(A)=g(A)$. Since $g$ is continuous at $A$, it follows that the restriction of $f$ to the closed set $\mathscr{A}$ is continuous at $A$. Hence, the restriction of $R$ to the closed set $\mathscr{A}$ is continuous. Thus, to complete 
the proof that $R$ is continuous, it will suffice to show that if $A \in \mathscr{A}$ and $\left\{A_{n}\right\}_{n=1}^{\infty}$ is a sequence in $2^{X}-\mathscr{A}$ such that $A_{n} \rightarrow A$, then $R\left(A_{n}\right) \rightarrow R(A)$. Let $\varepsilon>0$. For $A \in \mathscr{A}$, we will consider three distinct cases.

Case I. Suppose that $p \in A$ and $A \not \subset L_{0}$. Since $f$ is continuous at $A$, there exists $\delta>0$ such that if $B \in 2^{X}$ and $\rho(A, B)<\delta$, then $\rho(f(A), f(B))<\varepsilon$. Since $A_{n} \rightarrow A$, there exists $n_{1} \in \mathbf{Z}^{+}$such that $n \geqslant n_{1}$ implies $\rho\left(A_{n}, A\right)<\delta / 2$. For some $\theta>0$, $A \cap\left(L_{\theta}-\{p\}\right) \neq \varnothing$. It follows that there exists $n_{2} \in \mathbf{Z}^{+}$such that $n \geqslant n_{2}$ implies $\theta_{\max }\left(A_{n}\right)>\theta / 2$. Thus, since $r_{\text {min }}\left(A_{n}\right) \rightarrow r_{\text {min }}(A)=0$, there exists $n_{3} \in \mathbf{Z}^{+}$such that $n \geqslant n_{3}$ implies $\theta_{\max }\left(A_{n}\right) / r_{\text {min }}\left(A_{n}\right)>1$. Let $n \in \mathbf{Z}^{+}$such that $n \geqslant \max \left\{n_{1}, n_{3}\right\}$. Then

$$
R\left(A_{n}\right)=\alpha\left(A_{n}, 1\right)=f\left(A_{n}\right) \quad \text { and } \quad \rho\left(R\left(A_{n}\right), R(A)\right)=\rho\left(f\left(A_{n}\right), f(A)\right)<\varepsilon .
$$

Case II. Suppose that $p \in A$ and $A \subset L_{0}$. Let $\delta$ and $n_{1}$ be as in Case I. Since $r_{\text {min }}\left(A_{n}\right) \rightarrow r_{\text {min }}(A)=0$, there exists $n_{4} \in \mathbf{Z}^{+}$such that $n \geqslant n_{4}$ implies $r_{\min }\left(A_{n}\right)<$ $\delta / 2$. Let $n \in \mathbf{Z}^{+}$such that $n \geqslant \max \left\{n_{1}, n_{4}\right\}$. If $\theta_{\max }\left(A_{n}\right) \geqslant r_{\min }\left(A_{n}\right)$, then

$$
R\left(A_{n}\right)=\alpha\left(A_{n}, 1\right)=f\left(A_{n}\right) \text { and } \rho\left(R\left(A_{n}\right), R(A)\right)=\rho\left(f\left(A_{n}\right), f(A)\right)<\varepsilon .
$$

Now suppose that $\theta_{\max }\left(A_{n}\right) \leqslant r_{\text {min }}\left(A_{n}\right)<\delta / 2$. Let $\pi\left(A_{n}\right)=\left\{\left(r, \theta_{\text {min }}\left(A_{n}\right)\right) \mid(r, \theta) \in\right.$ $\left.A_{n}\right\}$. Since $\theta_{\min }\left(A_{n}\right) \leqslant \theta_{\max }\left(A_{n}\right)<\delta / 2, \rho\left(\pi\left(A_{n}\right), A_{n}\right)<\delta / 2$. Thus,

$$
\rho\left(\pi\left(A_{n}\right), A\right) \leqslant \rho\left(\pi\left(A_{n}\right), A_{n}\right)+\rho\left(A_{n}, A\right)<\delta / 2+\delta / 2=\delta .
$$

Hence, $\rho\left(f\left(\pi\left(A_{n}\right)\right), f(A)\right)=\rho\left(g\left(A_{n}\right), f(A)\right)<\varepsilon$. Since $\rho\left(\pi\left(A_{n}\right), A\right)<\delta$ and since $p \in A, \rho\left(\{p\} \cup \pi\left(A_{n}\right), A\right)<\delta$. Hence,

$$
\rho\left(f\left(\{p\} \cup \pi\left(A_{n}\right)\right), f(A)\right)=p\left(f\left(\{p\} \cup g\left(A_{n}\right)\right), f(A)\right)<\varepsilon .
$$

Since $\rho\left(f\left(\{p\} \cup g\left(A_{n}\right)\right), f(A)\right)<\varepsilon$ and $\rho\left(f\left(A_{n}\right), f(A)\right)<\varepsilon$,

$$
\rho\left(f\left(\{p\} \cup g\left(A_{n}\right)\right) \cup f\left(A_{n}\right), f(A)\right)<\varepsilon .
$$

Let $s \in I$. Then $g\left(A_{n}\right) \subset \alpha\left(A_{n}, s\right) \subset f\left(\{p\} \cup g\left(A_{n}\right)\right)$ or $f\left(\{p\} \cup g\left(A_{n}\right)\right) \subset$ $\alpha\left(A_{n}, s\right) \subset f\left(\{p\} \cup g\left(A_{n}\right)\right) \cup f\left(A_{n}\right)$ or $f\left(A_{n}\right) \subset \alpha\left(A_{n}, s\right) \subset f\left(\{p\} \cup g\left(A_{n}\right)\right) \cup$ $f\left(A_{n}\right)$. It follows that for each $s \in I, \rho\left(\alpha\left(A_{n}, s\right), f(A)\right)<\varepsilon$. Since $R\left(A_{n}\right) \in$ $\left\{\alpha\left(A_{n}, s\right) \mid s \in I\right\}$, we conclude that

$$
\rho\left(R\left(A_{n}\right), R(A)\right)=\rho\left(R\left(A_{n}\right), f(A)\right)<\varepsilon .
$$

Case III. Suppose that $p \notin A$. Since $\left\{A_{n}\right\}_{n=1}^{\infty} \subset 2^{X}-\mathscr{A}$ and since $A_{n} \rightarrow A$, it follows that $A \subset L_{0}-\{p\}$. Then $R(A)=f(A)=g(A)$. Since $h_{1}$ is uniformly continuous, there exists $\delta_{1}>0$ such that if $B_{1}, B_{2} \in C(X)$ and $s_{1}, s_{2} \in I$ such that $\rho\left(B_{1}, B_{2}\right)<\delta_{1}$ and $\left|s_{1}-s_{2}\right|<\delta_{1}$, then $\rho\left(h_{1}\left(B_{1}, s_{1}\right), h_{1}\left(B_{2}, s_{2}\right)\right)<\varepsilon$. Since $g$ is continuous at $A$, there exists $n_{5} \in \mathbf{Z}^{+}$such that $n \geqslant n_{5}$ implies $\rho\left(g\left(A_{n}\right), g(A)\right)<\delta_{1}$. Since $\theta_{\max }\left(A_{n}\right) \rightarrow \theta_{\max }(A)=0$ and $r_{\min }\left(A_{n}\right) \rightarrow r_{\min }(A)>0$, there exists $n_{6} \in \mathbf{Z}^{+}$ such that $n \geqslant n_{6}$ implies $\theta_{\max }\left(A_{n}\right) / r_{\min }\left(A_{n}\right)<\min \left\{\delta_{1} / 3,1 / 3\right\}$. Let $n \in \mathbf{Z}^{+}$such that $n \geqslant \max \left\{n_{5}, n_{6}\right\}$. Then

$$
\begin{aligned}
R\left(A_{n}\right) & =\alpha\left(A_{n}, \theta_{\max }\left(A_{n}\right) / r_{\min }\left(A_{n}\right)\right) \\
& =h_{1}\left(g\left(A_{n}\right), 3\left(\theta_{\max }\left(A_{n}\right) / r_{\min }\left(A_{n}\right)\right)\right) .
\end{aligned}
$$


Since $\rho\left(g\left(A_{n}\right), g(A)\right)<\delta_{1}$ and

$$
\left|3\left(\theta_{\max }\left(A_{n}\right) / r_{\min }\left(A_{n}\right)\right)-0\right|<3\left(\delta_{1} / 3\right)=\delta_{1}
$$

and since $h_{1}(g(A), 0)=g(A)$,

$$
\rho\left(h_{1}\left(g\left(A_{n}\right), 3\left(\theta_{\max }\left(A_{n}\right) / r_{\min }\left(A_{n}\right)\right)\right), h_{1}(g(A), 0)\right)=\rho\left(R\left(A_{n}\right), R(A)\right)<\varepsilon .
$$

The proof that $R$ is continuous is now complete. Since $C(X) \subset \mathscr{A}, R(A)=f(A)$ $=A$ for each $A \in C(X)$. Hence, $R$ is a retraction from $2^{X}$ onto $C(X)$.

Corollary 1. Let $F_{1}(X)=\{\{x\} \mid x \in X\}$. Then $F_{1}(X)$ is a retract of $2^{X}$.

Proof. Since $X$ is a smooth dendroid, it follows from Theorem 1 of [9] that $F_{1}(X)$ is a retract of $C(X)$. By Theorem $1, C(X)$ is a retract of $2^{X}$. Hence, $F_{1}(X)$ is a retract of $2^{X}$.

\section{REFERENCES}

1. J. T. Goodykoontz, Jr., Connectedness im kleinen and local connectedness in $2^{X}$ and $C(X)$, Pacific J. Math. 53 (1974), 387-397.

2. __ $C(X)$ is not necessarily a retract of $2^{X}$, Proc. Amer. Math. Soc. 67 (1977), 177-178.

3. Hyperspaces of arc-smooth continua, Houston J. Math. 7 (1981), 33-41.

4. Some functions on hyperspaces of hereditarily unicoherent continua, Fund. Math. 95 (1977), $1-10$.

5. J. D. Lawson, Applications of topological algebra to hyperspace problems, Lecture Notes in Pure and Appl. Math., No. 24, Marcel Dekker, New York, 1976, pp. 201-206.

6. S. B. Nadler, Jr., A characterization of locally connected continua by hyperspace retractions, Proc. Amer. Math. Soc. 67 (1977), 167-176.

7. __ Hyperspaces of sets, Dekker, New York, 1978.

8. Some problems concerning hyperspaces, Lecture Notes in Math., Vol. 375, Springer-Verlag, New York, 1974, pp. 190-197.

9. S. B. Nadler, Jr., and L. E. Ward, Jr., Concerning continuous selections, Proc. Amer. Math. Soc 25 (1970), 369-374.

10. M. Wojdyslawski, Retractes absolus et hyperespaces des continus, Fund. Math. 32 (1939), 184-192.

Department of Mathematics, West Virginia University, Morgantown, West Virginia 26506 